\title{
Editorial
}

Marc A. Reymond*

\section{Successful scientific quality review of Pleura and Peritoneum by PubMed Central}

https://doi.org/10.1515/pp-2018-0129

previously published online March 26, 2019

On October 22, 2018, De Gruyter Publisher was informed that the journal Pleura and Peritoneum passed successfully scientific quality review for PubMed Central (PMC).

The decision if a journal merits inclusion in PMC is made by the National Library of Medicine (NLM) based on its scientific and editorial character and quality. The scientific quality assessment for PMC focuses on the scientific rigor of the articles examined. NLM also looks for journals to demonstrate good editorial quality and elements that contribute to the objectivity and credibility of the content. PMC's scientific and editorial quality evaluation is a rigorous, multi-step process in which many factors are assessed (https://www. ncbi.nlm.nih.gov/pmc/pub/addjournal/\#sci-quality).

In issue 3/2018, Pleura and Peritoneum announced the founding of the International Society for the Study of Pleura and Peritoneum (ISSPP) in Paris. ISSP (www.isspp. org) is a non-profit international scientific organization aimed at relieving human suffering caused by pleural or peritoneal as well as other serosal disorders by improving research, innovation, education, training and patient care, as well as fostering interprofessional exchange. ISSPP is the first scientific organization focusing on the organs pleura, peritoneum, pericardium and other serosal surfaces in health and disease. ISSPP wants to encourage not only clinical research, but also basic and translational research on these organs, including embryology, anatomy, physiology, biochemistry, biophysics and other fundamental disciplines. Diseases covered by ISSPP include both benign and malignant pathologies and include gastrointestinal, gynecological and cardiothoracic locations. I am proud that, during the first meeting of its Executive Committee, ISSPP decided to make Pleura and Peritoneum the official journal of the society.
In September 2018, "P\&P", as we like to nickname our journal, published a special issue containing the abstracts of the 11th International Workshop of Peritoneal Surface Malignancies of the Peritoneal Surface Oncology Group International (PSOGI) in Paris. With more than 900 participants, this congress was the most successful meeting ever organized on the organs pleura and peritoneum.

Clearly, awareness and interest of the scientific and medical communities for the organs pleura and peritoneum - the "forgotten organs" - are increasing. Against this background and with the successful NLM scientific review, P\&P is the rising star in the field. P\&P publishes reviews, mini reviews, recommendations, educational and opinion papers. P\&P also covers research articles of experimental and clinical results, biochemical and anatomical findings. Finally, P\&P publishes translational research results.

P\&P is now a referenced, peer-reviewed, open-access journal. Since its launch three years ago, authors have not been charged for publication in P\&P. This generous policy, made possible by an unrestricted grant, will remain in place until the end of the year 2019.

On behalf of the Editorial Board and De Gruyter Publisher, I would like to renew my invitation to basic scientists, clinical researchers and physicians active in the field to take advantage of the unique opportunities offered by the Journal and to publish results of their organ-, disease- or therapy-focused research in Pleura and Peritoneum.

Marc A. Reymond, Department of Surgery and Transplantation, National Center for Pleura and Peritoneum, Comprehensive Cancer Center, Berlin, Germany; University Hospital, Hoppe-Seyler-Str. 3, 72076 Tübingen, Germany, E-mail: marc.reymond@med.uni-tuebingen.de 